\title{
Management of groin pain in athletes
}

\author{
Peter A Fricker
}

Groin pain is a frustration for both patient and therapist. Whilst there has been much discussion on this subject in the literature over recent years, there has been little real progress in defining simple diagnoses and rapid and effective treatments for the range of conditions that currently purport to produce groin pain.

This discussion describes the approach taken by the author to the management of groin pain in athletes and considers the patterns of pain, biomechanical factors, anatomical structures, and diagnostic tests, clinical and technical, that are all relevant. Not all these tests are proven, and much of the understanding of groin pain is empirical.

Even when a diagnosis has been made, treatment is not easy because of the difficulty of facilitating the healing of the many anatomical structures involved, and because so many modalities and methods are unproved.

\section{Method}

Computerised literature searches were performed for this review using Medicine and Sport Discus databases, and the period 1990-97 (February) was specified. Combinations of keywords were used to identify papers on groin pain in atheletes; these keywords included symphysis pubis, inguinal canal, groin pain, osteitis pubis, conjoint tendon, hernia, adductor, stress fracture, footballers, athletes, injury, and imaging.

The searches were not limited to English publications or to sports medicine publications. Additionally, the author's own collection of papers and personal correspondence on the subject was used. Many of these had been collected for a previous publication. ${ }^{1}$

References used were selected on each paper's individual contribution to understanding the nature, pathomechanics, and management of chronic groin pain in athletes. Outcomes of management protocols were especially considered.

Medicine, Australian

Institute of Sport,

Belconnen, Australia

P A Fricker

Correspondence to: Professor P A Fricker, Department of Sports Medicine, Australian Institute of Sport, PO Box Institute of Sport, PO
176, Belconnen, ACT, Australia 2617. helps us to list probable causes of pain and ability over possible causes. Understanding sport techniques is also vital, as, for example, a soccer player will sustain different injuries from a hurdler.
Groin pain is a vague description, hence the challenge. Clinically, the groin is hard to define. It involves the area where the abdomen meets the legs and includes the area of the crutch or perineum. Groin pain may thus present in the lower rectus abdominis musculature, the inguinal region, the area of the symphysis pubis, the upper adductors of the thigh, or the scrotum.

Groin pain may originate from muscles, tendons, bones, bursae, fascial structures, nerves, and joints. It may present acutely or otherwise, and may arise from more than one source.

The pattern of pain is important. Pain that emanates from the area of the symphysis pubis and radiates into the lower rectus abdominis, the upper adductors, and the scrotum is probably from the symphysis and suggests osteitis pubis. ${ }^{1}$ This pain may be unilateral or bilateral, may involve one or more sites from time to time, and may present acutely, or, more often, subacutely or by gradual onset. ${ }^{2}$ It typically presents during or after kicking and running, and is seen most often in footballers (soccer and Australian football players) where limitation of rotation of the hip is thought to be a contributing factor. ${ }^{3}$ This limitation is believed to transfer stress (either by shearing or distraction) across the symphysis and thus contributes to disruption of this fibrocartilaginous joint.

Some patients report a painful clicking at the symphysis on certain movements (such as rolling over in bed) ${ }^{4}$ but this symptom appears to be relatively uncommon. It may reflect instability of the symphysis, which is often seen on $x$ ray (the "flamingo view"), discussed below.

Local tenderness of the symphysis and loss of (particularly internal) rotation of the hips are the hallmark signs of osteitis pubis, and a bone scan (technetium-99m ( ${ }^{99 \mathrm{~m}} \mathrm{Tc}$ )) typically shows uptake on the delayed views of either or both margins of the symphysis. Plain radiography of the symphysis shows characteristic changes of erosion of one or both margins, with widening of the cleft, particularly at the lower end. The "flamingo view" is so called because the patient stands first on one leg and then on the other during $x$ ray to ascertain any shift across the symphysis. Movement across the joint of greater than $2 \mathrm{~mm}$ is significant ${ }^{5}$ and heralds pubic instability. This is not inevitably a sequel of osteitis pubis and may be asymptomatic.

Imaging of the symphysis with magnetic resonance imaging (MRI) is not well described 
in the literature, but evident widening of the cleft and (in some cases) posterior erosion of the pubic bodies at the margins of the joint has been observed in the few MRI studies made available. MRI of the symphysis in cases of peripartum rupture have been described, ${ }^{6}$ and changes include effusions and haemorrhage within the secondary clefts of the symphyseal cartilage and ligaments, with preservation of the surrounding ligaments of the joint. As there appears to be a subgroup of athletes with osteitis pubis who have a pre-existing obstetric or gynaecological history, ${ }^{1}$ similar changes may also been seen in the athletic context, particularly if the condition has presented acutely. This is an interesting area for further research.

A lesion of the conjoint tendon is better described as a posterior wall defect at the medial end of the inguinal canal. It is also often seen in footballers (of all codes) and is therefore commonly associated with osteitis pubis. ${ }^{2}$ Pain from a posterior wall defect ("conjoint tendon tear") typically appears local to the lesion, but may involve the lower rectus abdominis and the affected inguinal canal more diffusely. It does not appear to radiate to the scrotum (as is the case for osteitis pubis).

There is local pain and tenderness, which is emphasised if the patient half sits up while local pressure is maintained over the area of the conjoint tendon. There may also be a palpable small direct inguinal hernia at the site (hence the names "sports hernia" and "incipient inguinal hernia" given to this condition). ${ }^{7}$ Posterior wall defects of the medial inguinal canal (incipient hernias) may be bilateral.

Investigation is difficult, but some promising work is being carried out with ultrasonography ${ }^{8}$ which can demonstrate the defect in the medial posterior inguinal wall under real time (dynamic) imaging. "Herniography" by peritoneal imaging has also proven very useful, ${ }^{9}$ but this technique is not without morbidity and is technically demanding. When performed it can demonstrate filling defects in the posterior inguinal wall and can confirm that hernia repair in an athlete is warranted.

Adductor strain is relatively easy to diagnose. The patient usually provides a clear history of pain at the site of the lesion (usually adductor longus) associated with activities involving rapid adduction of the thigh-for example, kicking across the body, which may present

Table 1 Common causes of groin pain in athletes

Osteitis pubis

Incipient direct inguinal hernia (sportsman's hernia, conjoint tendon lesion)

Adductor muscle strain

Adductor tendinopathy

Stress fracture inferior pubis ramus

Osteomyelitis of pubic ramus or symphysis

Ilioinguinal neuropathy

Obturator neuropathy

Iliopsoas muscle strain (and/or bursitis)

Rectus femoris strain

Perthes' disease, avascular necrosis of femoral head

Slipped femoral epiphysis

Stress fracture neck of femur

Synovitis, osteoarthritis of hip joint

Iliolumbar ligament lesion

Sacroiliac ligament lesion

Spine pathology (radicular neuropathy L1 or L2, disc pathology L4-L5, lumbar zygapophysial joint pathology) acutely or by gradual onset, and which tends not to radiate much beyond the area of injury. ${ }^{10}$ Importantly, the clinician must decide whether the injury affects the tenoperiosteal attachment of the upper adductor or involves the musculotendinous junction or muscle belly proper. This distinction is necessary because management varies. Local tenderness and pain on resisted adduction confirm the diagnosis. Ultrasound imaging can be useful in delineating the lesion, as can computed tomography (CT) scanning or MRI, but these are not usually necessary.

Other causes of groin pain include stress fracture of the (usually inferior) pubic ramus ${ }^{11}$ and osteomyelitis of the anterior bony pelvis. ${ }^{12}$

Stress fractures typically present as gradually evolving pain and tenderness at the site of the fracture. Most often the inferior ramus is involved, and the condition is seen in female marathon runners in particular. ${ }^{11}$ As with all stress fractures, the cause is repetitive physical activity and the onset is related to periods of heavy training or competition.

There is local tenderness on examination (which may be nauseating in intensity), and often the patient may complain of pain when standing on one leg (of the ipsilateral side to the lesion). ${ }^{12}$ Running or jumping may, of course, be difficult. ${ }^{99 \mathrm{~m}} \mathrm{Tc}$ bone scan confirms the diagnosis readily in most cases, whereas plain radiography may be unhelpful. ${ }^{11}$

Osteomyelitis is reported to be a rare condition in healthy athletes, ${ }^{12}$ but its occurrence demands prompt recognition and management. Osteomyelitis of the symphysis is manifested by severe bony pain, local tenderness, and the development of systemic symptoms such as fever, malaise, and lassitude. Pain may radiate across the symphysis and mimic the bilateral pain of osteitis pubis. Weight bearing becomes difficult. Diagnosis is confirmed by finding positive blood cultures of Staphylococcus aureus in particular or a culture of pathogenic organisms obtained by needle aspiration or open biopsy. In the early stages the patient may be active, and erythrocyte sedimentation rate, bone scan, and radiography may all be unhelpful.

Of recent interest has been neurogenic groin pain. Lovell ${ }^{2}$ discusses the clinical presentation of ilioinguinal neuralgia in a series of athletes with groin pain, and noted that the diagnosis was made on finding pain in the area over the iliac fossa, tenderness at the point in the anterior abdominal wall where the nerve pierces the musculature (near the anterior superior iliac spine), and that injection of $2-5 \mathrm{ml}$ local anaesthetic at this site relieved the pain.

Obturator neuropathy has also been described as a cause of chronic groin pain in athletes. ${ }^{13}$ This diagnosis relies on noting exercise induced medial thigh pain over the area of the adductors, particularly after kicking and twisting. There may also be adductor muscle weakness and/or paraesthesia of the medial thigh after exercise. Electromyography demonstrates chronic denervation changes in the adductor muscles and the lesion is thought to result from nerve compression by fascial 
entrapment of the obturator nerve where it enters the thigh (at the adductor brevis).

Referred pain, as radicular neuropathy at the level of L1 or L2 in particular, may manifest as groin pain. Macnab ${ }^{14}$ suggests that posterior joint damage produced by degenerative changes of the L4-L5 disc may also produce pain referred to the groin, and more recently Bogduk and Twomey ${ }^{15}$ cite research that has shown that noxious stimulation of lumbar zygapophysial joints can cause referred pain in various regions of the lower limbs, including the groin.

Bursitis, particularly of the iliopsoas muscle of the hip, may also produce groin pain in athletes. ${ }^{16}$ This structure is irritated by repeated activity involving hip flexors and may therefore be a problem for runners, jumpers, hurdlers, and footballers. The diagnosis of iliopsoas bursitis may be extended to include inflammation of the iliopsoas muscle, or, if symptoms are acute, a tear or strain of this muscle. Inflammation of these structures produces a deep groin or anterior hip pain, which the patient finds difficult to localise and the clinician finds frustrating because of the lack of a point of deep tenderness in many cases. Resisted hip flexion may reproduce the symptoms, and a lunge whereby the affected groin and hip are forced into an extended position may also be painful. Ultrasound examination of deep hip structures usually produces disappointing results, despite good technical procedures. CT scanning or MRI may have something to offer.

Rectus femoris strain is easily diagnosed because of this muscle's relatively superficial location and because the history of pain and its whereabouts are usually quite definite. Local tenderness, irritability of muscle function (with or without evident weakness), and confirmation of the lesion by ultrasonography if needed, all make the diagnosis. Intramuscular tears can be associated with significant bleeding (and the

Table 2 Investigation of groin pain in athletes: selection of diagnostic tests

\begin{tabular}{ll}
\hline Test & Diagnosis \\
\hline Plain $x$ ray & Established osteitis pubis \\
& Stress fracture (later stages) \\
Osteomyelitis (later stages) & Slipped femoral epiphysis (epiphysiolysis) \\
& Osteoarthritis \\
& Osteitis pubis \\
${ }_{99 m}$ Tc MDP bone scan & Stress fracture \\
& Osteomyelitis \\
& Synovitis (occasionally bursitis) \\
& Avascular necrosis \\
& Sacroilitis \\
& Tenoperiosteal lesion \\
& Muscle tear \\
& Muscle tear, haematoma \\
& Inguinal hernia \\
Ultrasound scan & Bursitis (occasionally) \\
& Ilioinguinal neuropathy \\
Nerve conduction studies & Obturator neuropathy \\
Peritoneal radiography ("herniography") & Inguinal hernia \\
CT scan/MRI & Disc pathology \\
& Radicular lesion \\
& Osteitis pubis \\
& Other bone and soft tissue lesions as above \\
&
\end{tabular}

risk of calcification or myositis ossificans), and thorough evaluation of the extent of any injury to this muscle is warranted.

Hip joint pathology may present as exercise related groin pain. The differential diagnosis includes Perthes' disease and slipped femoral (capital) epiphysis in the young, synovitis of the hip at all ages, and stress fracture of the neck of femur, osteoarthritis (or osteoarthrosis) and avascular necrosis of the head of femur in the older patient. The diagnosis depends on a careful history and examination. Overuse contributes to the development of stress fracture and synovitis in particular, while osteoarthritic change is seen in those who have participated in vigorous sports, such as squash, or contact sports, such as football or rugby. Perthes' disease, slipped epiphysis, and avascular necrosis of the head of femur are notoriously insidious in onset and may only be discovered late, after the development of permanent changes. The universal caution to beware the child or adolescent who walks with a limp and complains of leg or knee pain applies.

Appropriate clinical examination includes assessment of rotation of the hips, leg length discrepancy, and gait, supported by imaging of the joint by plain radiography, CT scanning, or MRI together with ${ }^{99 \mathrm{~m}} \mathrm{Tc}$ bone scan. Synovitis of the hip may show diffuse isotope uptake on delayed views only, in the presence of normal radiography. Other lesions show morphological changes of the head of the femur and hip joint that are characteristic and well described. Stress fracture of the neck of femur and avascular necrosis may be discovered by changes on bone scan or MRI early in their development, with radiographic changes evident at later stages.

Posterior pelvic structures such as the iliolumbar ligament and posterior sacroiliac ligaments can also produce groin pain. Lesions of these structures produce local pain, which radiates into the groin and upper adductor area, as well as down the lateral thigh and posteriorly down the leg in the case of sacroiliac ligament lesions. ${ }^{17}$ Diagnosis is made on an appropriate history of low back and/or sacroiliac pain, together with local tenderness of involved ligamentous structures. Local injection of xylocaine should relieve symptoms and confirm the diagnosis.

Other causes of groin pain include pelvic, gynaecological, and urinary tract conditions. Common conditions occur commonly, and athletes are prone to the same range of ills as the rest of the population.

\section{Management}

Once the diagnosis has been made, there are useful steps in management for each clinical situation.

Osteitis pubis is perhaps the most difficult of the groin problems. The following regimen suggests a number of positive measures which may assist the affected athlete's outlook as much as their recovery. Firstly, osteitis pubis is a self-limited disease and every patient should expect to get better. Secondly, it is as yet unclear whether continued activity delays 
recovery, and so, at least for now, patients can be encouraged to maintain activity for fitness, such as swimming, cycling, and rowing, while avoiding those activities that are painful or that may worsen the condition (or delay its recovery) such as vigorous kicking or lots of running. Running in deep water ("pool running") may be a useful substitute for athletes wanting to maintain running fitness.

Improving flexibility of the hips (especially in rotation) is vital, as is correcting any limitation of movement in the sacroiliac or lumbosacral joints (which promotes excess movement at the symphysis).

Attention to muscle strength about the hips and pelvis and to biomechanical abnormalities of the lower limbs is also recommended.

Wearing supportive shorts made of lined neoprene (or similar) is often useful, as the local warmth and mechanical support provided give comfort, if nothing else.

Medication is less important than for other conditions. A short trial of non-steroidal anti-inflammatory drugs may identify those patients who will benefit from longer courses, but in any event the average time to recovery is nine to ten months.

Corticosteroid treatment (either by injection or orally) could be counterproductive. Corticosteroids are catabolic, and there is a potential risk of loosening the symphysis by use of these agents. Corticosteroid injection to the symphysis of athletes in the acute phase (less than two weeks) of osteitis pubis may hasten recovery, ${ }^{18}$ but larger studies need to be carried out to confirm this finding.

Lesions of the conjoint tendon, including direct inguinal hernias, do not appear to recover in athletes who continue their athletic activity. There is no doubt that surgery is effective for such patients with return to activity in about six weeks. ${ }^{1920}$ There is also the question of whether repair of an asymptomatic posterior wall defect should be performed for those who have been investigated for groin pain and for whom such an incidental finding has been reported. This may occur particularly in athletes with one symptomatic lesion but with bilateral defects evident radiographically. If repair is advised for the symptomatic lesion, then bilateral repair is probably appropriate, given the natural history of the condition.

Adductor strain is treated differently depending on the site of the lesion, either at the tenoperiosteal attachment or within the muscle (the musculotendinous junction or muscle belly). Acute strain at the tenoperiosteal attachment should be rested until pain and local tenderness have settled, with gentle stretching and strengthening to follow over a period of weeks. Running or sprinting should be encouraged as symptoms permit, with cutting (changing direction rapidly) and kicking introduced towards the end of recovery.

Muscle strain within the belly or at the musculotendinous junction can be managed more aggressively, provided bleeding has been stopped and the risks of muscle haematoma, calcification, or myositis ossificans have been addressed appropriately. Stretching, strength- ening, and return to activity follow standard practice guidelines for the management of muscle injury. ${ }^{21}$

Stress fractures of the inferior pubic ramus settle over two to five months with rest from running. ${ }^{22}$ Stress fracture of the neck of femur demands prompt attention, vigilance, and caution. Stress fracture of the upper cortex of the neck of femur requires immediate non-weightbearing (bed rest) and orthopaedic attention to guard against the risk of complete fracture, displacement, and aseptic necrosis of the femoral head. Stress fracture of the inferior cortex requires non-weight-bearing on crutches for three to four weeks, followed by gradual resumption of full weight-bearing over the ensuing weeks.

Osteomyelitis is managed according to standard principles and involves the appropriate use of specific antibiotics, both intravenously and orally, supported by bed rest as necessary and analgesics. Surgery may be required where conservative measures fail. Return to sport is usually delayed and averages about five months. ${ }^{12}$

Nerve entrapments are relieved by appropriate surgical decompression (for a definitive cure). This applies particularly to the obturator neuropathy described above, whereby the thick fascia overlying the adductor brevis anteriorly is divided. ${ }^{13}$ Ilioinguinal nerve entrapment may be successfully managed by local infiltration with corticosteroid.

The management of spinal conditions that contribute to groin pain is beyond the scope of this paper but there are very many excellent texts available on this subject.

Iliopsoas muscle strain and associated bursitis are managed by anti-inflammatory medication and modalities, together with appropriate stretching and strengthening of the iliopsoas muscles. Local injection of corticosteroid can be tried but the difficulty is placing the injection at the site of injury. Certainly, bursitis should respond quickly to corticosteroid injection. Return to activities such as kicking and lunging should be cautious and promoted carefully within the limits of pain.

Strain of the rectus femoris is managed along the principles outlined above, with similar precautions applied to return to kicking or jumping movements. Haematoma(ta) within the quadriceps complex must be managed carefully to minimise rebleeding and/or calcification (myositis ossificans). This implies appropriate anti-inflammatory modalities and the avoidance of premature stretching and vigorous massage in particular.

Hip joint pathology should involve early referral to an orthopaedic surgeon for the management of Perthes' disease, avascular necrosis of the head of the femur, slipped femoral epiphysis, and stress fracture of the neck of femur. All of these conditions are prone to debilitating sequelae and should never be underestimated. Conservative management can be tried in many of these conditions but the decision to intervene surgically is critical and not for the inexperienced or untrained practitioner. 
Synovitis of the hip can be managed with rest and anti-inflammatory modalities, but the clinician must always have a diagnosis in mind. Synovitis of the hip is often secondary to a disease process and not necessarily an overuse or benign self-limiting condition. The management of osteoarthritis of the hip depends on the clinical state of the joint and can vary from simple measures of appropriate symptom relief and rehabilitative exercise (for strength and flexibility of the joint), to surgical intervention and joint replacement. It may be necessary to involve a rheumatologist and orthopaedic surgeon in such cases.

Management of posterior pelvic problems such as iliolumbar ligament sprain and posterior sacroiliac ligament sprain depends upon attention to causative factors such as trauma and/or faulty pelvic mechanics, together with the traditional forms of therapy including mobilisation, soft tissue massage, and antiinflammatory modalities.

\section{Summary}

Groin pain is a difficult clinical problem because of the variety of conditions that are potentially responsible. Much of the "theory" of groin pain is just that, theory, and much needs to be done to document the pathomechanics and symptomatology of this anatomical region.

Notwithstanding the above, clinicians should be comfortable in the knowledge that they can provide relief in most cases and a cure in more than a few.

The recommended diagnostic approach is anatomical, supported by judicious selection of diagnostic imaging techniques. Management then comprises a considered approach to functional recovery, allowing time to heal, regain strength, and restore mobility. Patients should be reminded that there are no short cuts and precipitate return to sport is not worth the risk in most cases.

1 Fricker PA, Taunton JE, Ammann W. Osteitis pubis in athletes. Infection, inflammation or injury? Sports Med letes. Infection,

2 Lovell G. The diagnosis of chronic groin pain in athletes: a review of 189 cases. Australian fournal of Science and Medicine in Sport 1995;27:76-9.

3 Williams JGP. Limitation of hip joint movement as a factor in traumatic osteitis pubis. Br F Sports Med 1978;12:129-33.

4 Harris NH, Murray RO. Lesions of the symphysis in athletes. $B M \mathcal{F} 1974 ; 4: 211-14$

5 Walheim G, Olerud S, Ribbe T. Mobility of the pubic symphysis: measurements by an electromechanical method. Acta Orthop Scand 1984;55:203-8.

6 Jurzel RB, Au AH, Rooholamini SA, Smith W. Magnetic resonance imaging of peripartum rupture of the symphysis resonance imaging of peripartum rupt

7 Hackney RG. The sports hernia: a cause of chronic groin pain. Br f Sports Med 1993;27:58-62.

8 Read JW, Crichton KJ, Orchard JW, Flahive SR. Sports hernia. In: Proceedings of the 26th Annual Scientific Meeting of the Australasian Society for Ultrasound in Medicine (abstract) 1996:69.

9 Gullmo A. Herniography. World f Surg 1989;13:560-8.

10 Karlsson J, Sward L, Kalebo P, Thomee R. Chronic groin injuries in athletes. Recommendations for treatment and rehabilitation. Sports Med 1994:17:141-8.

11 Noakes TD, Smith JA, Lindenberg GM, Wills CE. Pelvic stress fractures in long distance runners. Am $¥$ Sports Med stress fractures

12 Karpos PA, Spindler KP, Pierce MA, Shull HJ. Osteomyelitis of the pubic symphysis in athletes: a case report and literature review. Med Sci Sports Exerc 1995;27:473-8.

13 Bradshaw C, McCrory P, Bell S, Brukner P. Obturator neuropathy. A cause of chronic groin pain in athletes. $A m^{f}$ Sports Med. In press.

14 Macnab I. Backache. Baltimore: Williams and Wilkins, 1979:91.

15 Bogduk N, Twomey LT. Clinical anatomy of the lumbar spine. 2nd ed. Melbourne: Churchill Livingstone, 1991:154.

16 Peterson L, Renstrom P. Sports injuries. Their prevention and treatment. Methuen Australia, 1986:265.

17 Hackett GS. Ligament and tendon relaxation treated by prolotherapy. 3rd ed. Springfield, IL: CC Thomas, 1958:27-8.

18 Holt MA, Keene JS, Graf BK, Helwig DC. Treatment of Holt MA, Keene JS, Graf BK, Helwig DC. Treatment of
osteitis pubis in athletes. Results of corticosteroid injecosteitis pubis in athletes. Results of cort
tions. Am $\mathcal{F}$ Sports Med 1995;23:601-6.

19 Polglase AL, Frydman GM, Farmer KC. Inguinal surgery for debilitating chronic groin pain in athletes. Med $\mathfrak{F}$ Aust 1991;155:674-7.

20 Malycha P, Lovell G. Inguinal surgery in athletes with chronic groin pain: the "sportsman's" hernia. Aust $N Z \mathcal{F}$ Surg 1992;62:123-5.

21 Crichton KJ, Fricker PA, Purdam C, Watson AS. Injuries to the pelvis and lower limb. Science and medicine in sport. 2nd ed. Melbourne: Blackwell Science, 1995:434

22 Pavlov M, Nelson TL, Warren RF, Torg JS, Burstein AH. Stress fractures of the pubic ramus. $\mathcal{f}$ Bone foint Surg $[\mathrm{Am}]$ Stress fractures of 